\title{
NOTA
}

\section{ALERO MOLINA I. FUNCIONALIDAD Y CRONOLOGÍA DE UN REPARO PEQUEÑO DURANTE EL PLEISTOCENO FINAL Y EL HOLOCENO TARDÍO, TANDILIA ORIENTAL (ARGENTINA)}

\author{
ALERO MOLINA I. FUNCTIONALITY AND CHRONOLOGY OF A SMALL ROCKSHELTER \\ (FINAL PLEISTOCENE AND LATE HOLOCENE), EASTERN TANDILIA (ARGENTINA)
}

Diana Leonis Mazzanti ${ }^{*}$ y Gustavo Federico Bonnat ${ }^{* *}$

Fecha de recepción: 18 de enero de 2021

Fecha de aceptación: 29 de marzo de 2021

\section{INTRODUCCIÓN, ANTECEDENTES Y PROBLEMAS DE INVESTIGACIÓN}

Los proyectos que desarrolla el grupo de investigadores del Laboratorio de Arqueología Regional Bonaerense de la Universidad Nacional de Mar del Plata (LARBO-UNMDP) consolidaron líneas de trabajo multidisciplinares a escala microrregional (Tandilia oriental). Estas permitieron indagar variadas problemáticas características de la arqueología de la pampa húmeda, como por ejemplo, las transformaciones socioeconómicas ocurridas desde el poblamiento inicial, los distintos sistemas tecnológicos y de subsistencia característicos de cada período y las interacciones sociales pre y posconquista, entre otros temas (Politis y Madrid 2001; Mazzanti 2013; etc.). Cabe recordar que la puesta en valor de esta área de investigación se funda sobre escasos antecedentes arqueológicos, entre los que se encuentra la labor del geólogo A. Tapia (1937) y las prospecciones y excavaciones iniciadas por el arqueólogo J. Carbonari (1972). Estos investigadores descubrieron reparos rocosos y la riqueza, no solo del sector de estas sierras, sino también de los sitios pequeños como lo demostraron con Caverna Ojo de Agua y Cerro Valdés.

A partir de 1980 con el hallazgo del sitio Alero Molina I ${ }^{1}$ comenzó el desarrollo sistemático de las investigaciones en el borde oriental de las sierras de Tandilia, cuyos logros iniciales condujeron al registro de numerosos conjuntos arqueológicos en reparos rocosos (cuevas, abrigos y

\footnotetext{
* Laboratorio de Arqueología Regional Bonaerense, Universidad Nacional de Mar del Plata. E-mail: arqueolab@gmail.com

** Laboratorio de Arqueología Regional Bonaerense, Universidad Nacional de Mar del Plata. E-mail: fbonnat@hotmail.com
} 
aleros) (figura 1). Estos tipos de yacimientos dentro de cavidades rocosas presentan diferentes calidades de preservación de los depósitos estratificados debido a la mayor o menor exposición a los agentes naturales, biológicos y humanos (Martínez y Mazzanti 2017); no obstante, se convierten en espacios donde se conservan evidencias paleoambientales y culturales significativas. La conformación de un corpus empírico numeroso y variado en esta área de investigación respalda la reconstrucción de la secuencia arqueológica de larga duración (ca. 13.000 años cal. AP). Ésta, demostró el uso humano del ambiente serrano en coherencia con las problemáticas analizadas en otros sectores y/o ambientes de la región pampeana (Politis y Madrid 2001; Martínez 2006; Mazzanti 2013; entre otros).

Como se mencionó, el Alero Molina I fue el primer sitio arqueológico descubierto por uno de los autores (DM) que estimuló el inicio de las prospecciones sistemáticas del macizo serrano de La Vigilancia. Pocos meses después se localizó el sitio Cueva Tixi (1981) y una sucesión de nuevos sitios en reparos rocosos. Como resultado de esa labor de campo, el Alero Molina I no fue considerado para su estudio inmediato, debido a la riqueza arqueológica observada en los otros reparos que concentraron las investigaciones durante varias décadas (Mazzanti 2013). Esa trayectoria confluyó en varias propuestas y modelos explicativos sobre la dinámica social indígena durante los diferentes períodos de la arqueología pampeana (Mazzanti 2007; Bonnat 2020; Donadei Corada 2020). Otras investigaciones doctorales estuvieron centradas en la caracterización de aspectos paleoambientales y paleontológicos sobre la base de registros proxies y faunísticos de los sitios del área (Colobig 2011; Fernández Osuna 2019).

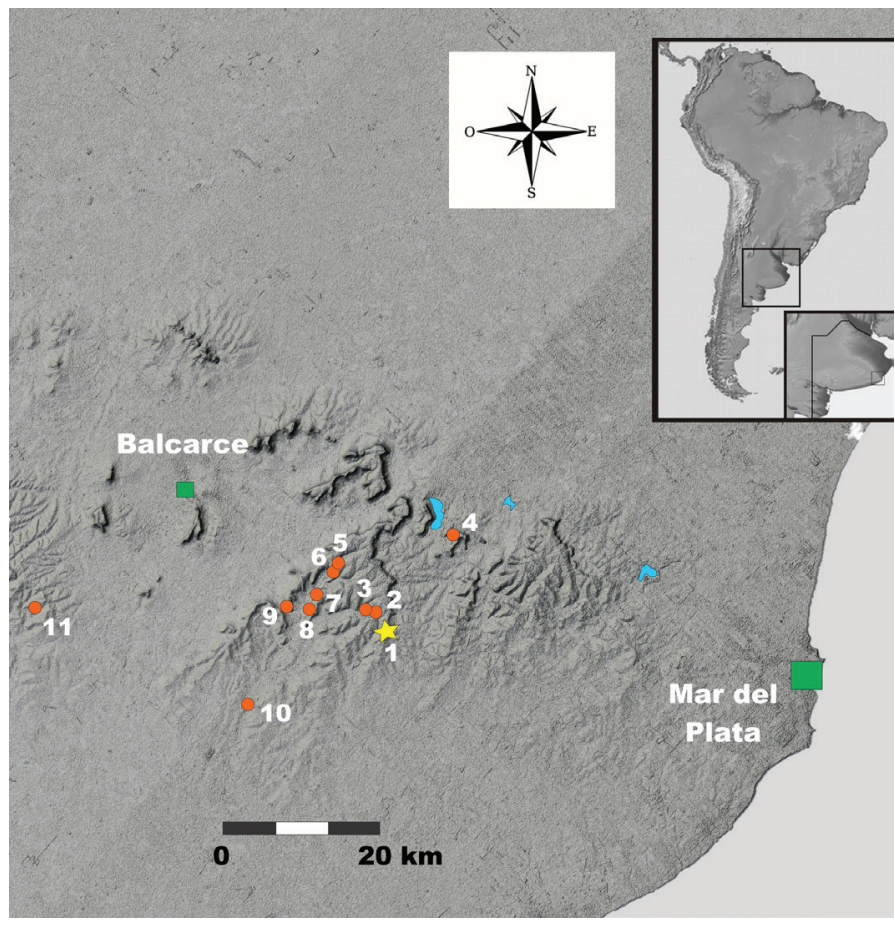

Figura 1. Mapa del área de Tandilia oriental con la localización de los sitios arqueológicos estudiados:

1. Alero Molina I; 2. Cueva Tixi; 3. Cueva Alí Mustafá; 4. Cueva La Brava; 5. Alero El Mirador;

6. Abrigo Los Pinos; 7. Abrigo La Grieta; 8. Cueva Burucuyá; 9. Cueva El Abra; 10. Localidad arqueológica Amalia; 11. Localidad Arqueológica Lobería I 
En este contexto de producción científica se retomó la investigación de los reparos pequeños con el objetivo de responder algunas cuestiones vinculadas a la selección de esos emplazamientos y a las funciones que tuvieron estos paraderos transitorios en el ordenamiento espacial del paisaje serrano (Mazzanti et al. 2019). Esta orientación toma la perspectiva general de la arqueología del paisaje (Criado Boado 1999) aplicada en otra contribución (Mazzanti y Bonnat 2013). En continuidad con esta línea, se plantearon varios problemas de investigación tales como: ¿por qué los grupos de cazadores-recolectores incorporaron estos pequeños paraderos serranos en sus circuitos de desplazamientos?, en otras palabras ¿qué factores pudieron determinar la ocupación de aleros con muy baja protección de los agentes naturales?, ¿fueron sitios estratégicos en los circuitos de tránsito por su alta visibilización local? Por lo expresado, esta contribución tiene como objetivo ofrecer los primeros resultados de las investigaciones en curso, exponiendo las características generales del alero, las nuevas cronologías y los aspectos generales de la tecnología lítica asociadas a dos períodos centrales de la arqueología pampeana.

\section{CARACTERÍSTICAS DEL ALERO MOLINA I}

El alero es una pequeña cavidad de aproximadamente $5 \mathrm{~m}^{2}$ bajo techo localizada en uno de los dos extremos más sur-orientales de la sierra de La Vigilancia (S3758'20" y O58²'98” a una altitud de 170 m s.n.m.), ubicado en el borde de mayor altura de ese sector serrano. El talud del alero está compuesto por bloques inclinados sobre la barda que condicionan el espacio y los movimientos, pero tiene la ventaja de hallarse a pocos metros de la cima extensa y plana (figura 2).

Los trabajos de campo tuvieron dos etapas, la inicial en 1980 con un sondeo $(40$ x $40 \mathrm{~cm})$ y la segunda con dos campañas de excavaciones (2016 y 2018) que alcanzaron la apertura de un área de $2 \mathrm{~m}^{2}$ (figura 3A). Se utilizó la técnica de decapados de unidades sedimentarias naturales con descripción de rasgos y/o elemento naturales y culturales. El reconocimiento geoarqueológico y los muestreos sedimentarios fueron realizados in situ por el doctor Gustavo Martínez (Instituto de Geología de Costa y Cuaternario -CIC-IMMyC-UNMDP-) y los resultados de laboratorio (granulometría, $\mathrm{pH}$, materia orgánica, etc.) aún están pendientes.

La génesis geológica de este sitio es concordante con la morfología estructural de los otros reparos rocosos de Tandilia oriental, todos fueron originados por la acción de procesos de meteorización sobre los planos de estratificación y diaclasas de las ortocuarcitas horizontales de la Formación Balcarce, los que actuaron formando diversos tipos de cámaras. Estos factores también incidieron en la composición sedimentaria, aportando materiales endógenos y exógenos por acción eólica, hídrica y de insolación. El conjunto de estos agentes contribuyó a la formación de eventos pedogenéticos en las entradas de los reparos rocosos (Gustavo Martínez comunicación personal).

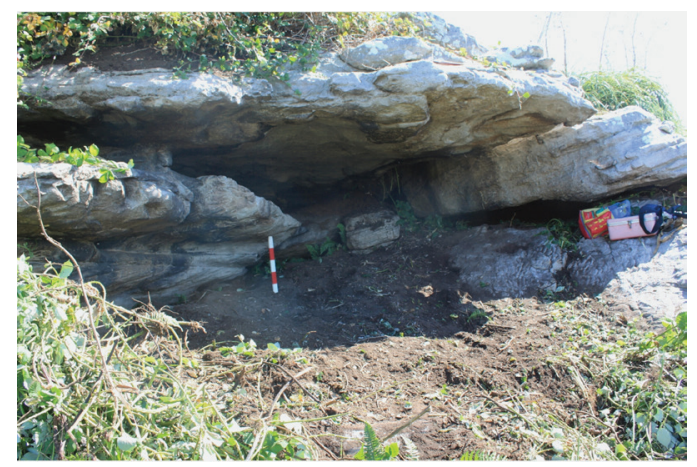

Figura 2. Vista del sitio arqueológico Alero Molina I 
Las características geoarqueológicas mencionadas se identificaron en el relleno del Alero Molina I cuyo espesor varía entre 0,40 y 0,60 m, lo que indica una secuencia sedimentaria del tipo condensada al formarse con tasas de sedimentación menores respecto a otras matrices (Martínez y Mazzanti 2017). Los estudios preliminares reconocen dos unidades estratigráficas (U1 y U2) con rasgos posdepositacionales (interface o discordancia erosiva y bioturbación) (figura 3 B y D). La inclinación que presenta parte del piso hacia la boca del reparo pudo favorecer el transporte hídrico de materiales livianos como sedimentos y carbones, por ello, se considera que la acción del viento y del agua fueron los procesos erosivos postdepositacionales que mayormente actuaron sobre la matriz sedimentaria, modelando las condiciones de su relleno, el registro estratigráfico y la menor preservación de carbón en sus depósitos.


Figura 3. Alero Molina I. A. plano de planta con indicación del sector excavado. Referencias: Las líneas de guiones refieren a la línea de gotera y a los contornos internos de bloques que afloran de los laterales y del piso de la cámara; B. Unidades estratigráficas (U1 y U2) de las cuadriculas B2-C2 con indicación de la Interface o discordancia erosiva (IE); C. Vista vertical de la Cuadricula C2 con clastos incluidos en la IE que afloran en los perfiles; D. Perfil Sur-oeste de la cuadricula C1, se observan dos rasgos de bioturbación y la IE

Los conjuntos arqueológicos se conservaron en dos unidades separadas por la discordancia erosiva de límite ondulado con rasgos de bioturbación y clastos chatos en planta dispuestos a la misma altura, los que se observan aflorando en los perfiles del área excavada (figura 3C). Este fenómeno de presencia de clastos planares incluidos en depósitos intermedios fue observado en otros reparos rocosos de la misma sierra que contienen matrices del Pleistoceno tardío-Holoceno. Los estudios geoarqueológicos determinaron la relación de estos clastos rocosos desprendidos de los techos de las cavidades, como productos de eventos paleoclimáticos del post-Hipsytermal (ca. 4500-5000 AP). Los sitios que contienen esas unidades y rasgos similares, brindaron dataciones que los ubican en el Holoceno medio: Cueva Tixi, Lobería I Sitio 1, Abrigo Los Pinos, Alero El Mirador, Abrigo La Grieta (Martínez 2007; Martínez y Mazzanti 2017; entre otros). En este contexto microrregional es posible proponer preliminarmente que esa discordancia tuvo su génesis en el Holoceno medio.

Con el fin de obtener cronologías de las dos ocupaciones humanas se procesaron dos muestras de carbón recuperadas en asociación con los materiales arqueológicos. Las dataciones se 
D. L. Mazzanti y G. F. Bonnat - Alero Molina I. Funcionalidad y CRonología de un ReParo PeQueÑo ...

realizaron por ${ }^{14} \mathrm{C}$ (AMS) en el Laboratorio de la Universidad de Arizona (USA), y calibrados con el programa Calib REV 8.2 (Stuiver et al. 2020) y la curva SHCal 20 (Hogg et al. 2020). Los resultados señalan que la ocupación humana registrada en la porción basal de la secuencia sedimentaria corresponde a la transición Pleistoceno-Holoceno. El segundo contexto se reconoció en un sector acotado de la unidad superior datado en Holoceno tardío (tabla 1).

Tabla 1. Dataciones radiocarbónicas del sitio Alero Molina I

\begin{tabular}{|c|c|c|c|c|c|}
\hline Código & Años ${ }^{\mathbf{1 4}} \mathbf{C}$ AP & Material & $\begin{array}{c}\text { Años cal. AP } \\
\text { (mediana) }\end{array}$ & $\begin{array}{c}\text { Rangos de años cal. } \\
\text { AP }(\mathbf{1} \text { sigma) }\end{array}$ & $\begin{array}{c}\text { Rangos de años cal. } \\
\text { AP }(\mathbf{2} \text { sigma })\end{array}$ \\
\hline AA108367 & $735 \pm 19$ & Carbón & 646 & $650-667$ & $634-671$ \\
\hline AA111628 & $10628 \pm 32$ & Carbón & 12627 & $12651-12687$ & $12606-12694$ \\
\hline
\end{tabular}

\section{CARACTERÍSTICAS GENERALES DE LOS CONJUNTOS LÍTICOS}

El conjunto total de materiales líticos hallados en el sitio está compuesto por 130 elementos. En el sondeo inicial y en las cuadriculas B2 y C1 se recuperaron artefactos líticos ( $\mathrm{N}=12)$ correspondientes al Holoceno tardío manufacturados con dos tipos de ortocuarcitas: Grupo Sierras Bayas (OGSB) y Formación Balcarce (OFB) (tabla 2; figura 4 A y B). Con respecto a las OGSB, las fuentes de abastecimiento se encuentran a unos $100 \mathrm{~km}$ de distancia del Alero Molina I y en el componente tardío están representadas por desechos de la talla. En cambio, las OFB son rocas que pueden ser obtenidas en las cercanías al sitio arqueológico y está representada en desechos de la talla y en un núcleo agotado (tabla 2).

Tabla 2. Conjunto lítico del sitio Alero Molina I. Clases artefactuales y materias primas identificadas

\begin{tabular}{|c|c|c|c|c|c|c|}
\hline \multirow[t]{2}{*}{ Ocupación } & \multirow[t]{2}{*}{ Rocas } & \multicolumn{4}{|c|}{$\begin{array}{c}\text { Clases artefactuales } \\
\mathbf{N}(\%)\end{array}$} & \multirow[t]{2}{*}{ Total } \\
\hline & & Desechos & Instrumentos & Microdesechos & Núcleos & \\
\hline \multirow{2}{*}{$\begin{array}{l}\text { Holoceno Tardío } \\
\qquad(\mathrm{N}=12)\end{array}$} & OFB & $4(80)$ & 0 & 0 & $1(20)$ & $5(41,66)$ \\
\hline & OGSB & $7(100)$ & 0 & 0 & 0 & $7(58,33)$ \\
\hline \multicolumn{2}{|c|}{ Subtotal } & $11(91,66)$ & 0 & 0 & $1(8,33)$ & $12(100)$ \\
\hline \multirow{9}{*}{$\begin{array}{c}\text { Transición } \\
\text { Pleistoceno- } \\
\text { Holoceno }(\mathrm{N}= \\
\text { 118) }\end{array}$} & Basalto & $1(100)$ & 0 & 0 & 0 & $1(0,84)$ \\
\hline & Caliza S. & $2(66,66)$ & 0 & $1(33,33)$ & 0 & $3(2,54)$ \\
\hline & Cuarzo & $5(62,5)$ & 0 & $3(37,5)$ & 0 & $8(6,77)$ \\
\hline & Diabasa & $1(50)$ & $1(50)$ & 0 & 0 & $2(1,69)$ \\
\hline & Indet. & $3(100)$ & 0 & 0 & 0 & $3(2,54)$ \\
\hline & Metac. & $1(50)$ & 0 & $1(50)$ & 0 & $2(1,69)$ \\
\hline & OFB & $49(92,45)$ & 0 & $2(3,77)$ & $2(3,77)$ & $53(44,91)$ \\
\hline & OGSB & $33(73,33)$ & $3(6,66)$ & $6(13,33)$ & $3(6,66)$ & $45(38,18)$ \\
\hline & Sílice & $1(100)$ & 0 & 0 & 0 & $1(0,84)$ \\
\hline \multicolumn{2}{|c|}{ Subtotal } & $96(81,35)$ & $4(3,38)$ & $13(11,01)$ & $5(4,23)$ & 118 \\
\hline
\end{tabular}

Referencias: OFB: ortocuarcitas de la Formación Balcarce; OGSB: ortocuarcitas del Grupo Sierras Bayas; Indet.: indeterminadas; Metac.: metacuarcitas. 
Los artefactos correspondientes a la transición Pleistoceno/Holoceno ( $\mathrm{N}=118)$ se hallaron en todas las cuadrículas del área excavada, señalando la mayor densidad artefactual, diversidad de clases y de materias primas representadas (tabla 2). Las rocas ingresadas en ese período al alero señalan la preferencia por el abastecimiento y uso de las ortocuarcitas, tanto locales (OFB) como regionales (OGSB) integradas en distintas clases artefactuales (tabla 2). Para el caso de la OFB los ocupantes del sitio seleccionaron preferentemente la variedad de grano medio-fino, reconocida en desechos de talla y en núcleos. Esta roca se encuentra en algunos bloques y estratos de la misma sierra, presentando mejor calidad para la talla respecto de la OFB de grano grueso. Algunos artefactos conservan corteza y presentan superficies de extracción en múltiples direcciones y también se la identificó en desechos y microdesechos de talla.

El análisis preliminar permitió identificar distintas clases artefactuales en OGSB que incluyen núcleos y fragmentos de núcleos, desechos de talla e instrumentos (tabla 2). Esta roca se presenta en una amplia diversidad de colores, entre los que se incluye el blanco, amarillo, rojo, rosa y algunas combinaciones de estos (figura $4 \mathrm{E} \mathrm{y} \mathrm{F),} \mathrm{aspecto} \mathrm{registrado} \mathrm{en} \mathrm{otros} \mathrm{contextos}$ arqueológicos del mismo período y del área.

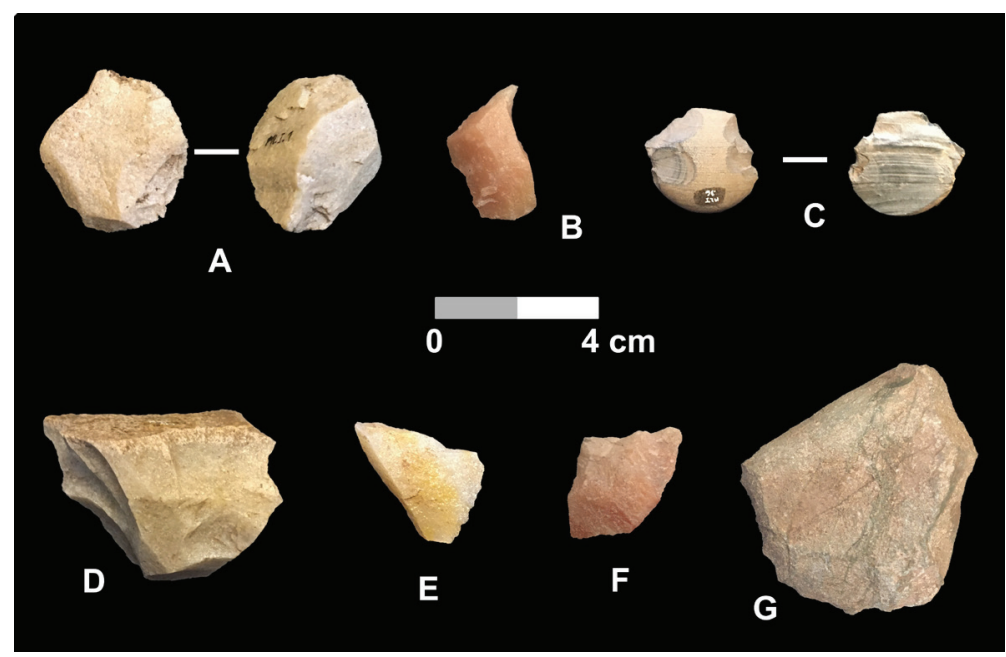

Figura 4. Artefactos líticos del sitio Alero Molina I. Componente Superior-U1-: A. núcleo agotado de OFB de grano medio-fino; B. instrumento compuesto (muesca con punta burilante) de OGSB color naranja. Componente Inferior-U2-; C. rodado de basalto con talla bipolar; D. núcleo de OFB de grano medio-fino; E. Fragmento de instrumento (filo natural con rastros complementarios) de OGSB color amarillo; F. lasca de OGSB color naranja; D. instrumento con filo denticulado de diabasa

Resulta significativa la presencia de otras materias primas (diabasa, metacuarcita, basalto, caliza silicificada y otras indeterminadas) (tabla 2; figura $4 \mathrm{G}$ ). Esta diversidad de tipos de rocas representa otro patrón recurrente observado en sitios tempranos de Tandilia oriental (Bonnat 2019, 2020). Estas materias primas proceden de distintas escalas espaciales y se presentan como desechos internos y microdesechos de la talla. Lo significativo de la presencia de estas variedades es la ubicación geográfica de sus fuentes de origen, las que incluyen el litoral marino donde se pudo obtener rodados costeros de basalto (figura 4 C) (Bonomo y Prates 2014) o escalas espaciales más amplias como en el caso de las metacuarcitas del sistema serrano de Ventania ( $c a$. $400 \mathrm{~km}$ del sitio) (Catella et al. 2020) y las calizas silicificadas de Entre Ríos y/o la República del Uruguay (ca. $500 \mathrm{~km}$ del sitio) (Bonomo y Blasi 2010; Flegenheimer et al. 2003; Loponte et al. 2010). 


\section{DISCUSIÓN Y CONCLUSIONES}

Los estratos horizontales de ortocuarcitas que conforman las sierras de la Formación Balcarce poseen características que favorecieron la génesis de numerosos reparos en las pendientes (Martínez 2007). Particularmente, en los macizos serranos mesetiformes donde, además, se destaca la importante red hídrica que contienen los valles internos, que fueron ámbitos de concentración de una valiosa biodiversidad utilizada por los cazadores-recolectores, no solo para la subsistencia, sino también para la producción de tecnologías. Un factor importante es la dimensión altitudinal de las cimas planas como recurso ventajoso para el dominio de la visibilización del paisaje, de los movimientos de animales y de grupos de cazadores-recolectores. Estas condiciones fueron propuestas en el modelo de movilidad y sistema de sitios estratégicos investigado en esta área (Mazzanti y Bonnat 2013; Mazzanti et al. 2019).

Esos macizos serranos se conformaron como verdaderos oasis ocupados por los cazadores-recolectores durante milenios. Sus cimas extensas y planas con laderas y faldeos que decrecen en altura hacia el sureste conectaron los valles longitudinales de gran accesibilidad, convirtiéndose en una geografía de tránsito, facilitadora de la movilidad e interacción al unir en tramos de fácil acceso y circulación las cuencas de ocupación (arroyo La Vigilancia y arroyo San Pedro). Los sitios pequeños emplazados en puntos geográficos de alta visibilización del paisaje fueron eslabones de esos circuitos, desempeñando un rol estratégico en la identificación de recursos naturales y movimientos sobre los territorios de sierras y llanuras adyacentes.

Los artefactos líticos atribuidos al Holoceno tardío presentan pocas piezas y si bien evidencian el uso de las dos variedades de ortocuarcitas, se observa que estas dos rocas fueron utilizadas prácticamente en las mismas frecuencias señalando la utilización efímera del alero. En contraste, el registro lítico de la ocupación inicial (transición Pleistoceno/Holoceno) se distingue del componente superior por la mayor diversidad de rocas incorporadas al sitio. Los ocupantes del alero durante ese período utilizaron rocas que fueron obtenidas en distintas escalas espaciales, las que alcanzan los $500 \mathrm{~km}$ de distancias del sitio Molina I. Esta información indica los amplios circuitos de movilidad y/o de interacción social que realizaron los primeros grupos de cazadores-recolectores que ocuparon este sector de la región pampeana. Nuevamente, se observan recurrencias en el patrón de aprovisionamiento de rocas con los otros sitios arqueológicos del área de estudio caracterizados como campamentos logísticos con ocupaciones breves o efímeras (e.g. Abrigo La Grieta, Cueva La Brava, Alero El Mirador, Amalia Sitio 2 y Cueva Alí Mustafá) y campamentos bases residenciales de actividades múltiples (e.g. Cueva Tixi, Abrigo Los Pinos y Cueva El Abra) (Bonnat 2019, 2020; Mazzanti et al. 2019). Asimismo, también la presencia de diversos tipos de roca fue propuesta en otras áreas de la región pampeana bonaerense (Flegenheimer et al. 2003; Martínez 2006; entre otros).

En síntesis, se propone que el Alero Molina I se configuró en un lugar utilizado durante el Pleistoceno final y Holoceno tardío como campamento de actividades restringidas por su función de sitio de avistamiento. Este sitio integró los circuitos de movilidad en la organización espacial ocurrida en cada período al situarse en un punto panorámico privilegiado desde el cual se alcanza un arco de visualización del paisaje de aproximadamente $270^{\circ}$, el que incluye el horizonte noroeste con la vista de las bardas serranas de La Vigilancia y El Volcán, sierra La Brava, Cerro Valdez y sectores del macizo de La Peregrina (figura 5A). Hacia el este y sudeste se divisa la extensa llanura y lomas que finalizan en el litoral atlántico (figura 5B) y continúa el domino visual de la llanura sur, constituyéndose este alero en un mojón o paradero estratégico en la cuenca panorámica sur-oriental de Tandilia. 


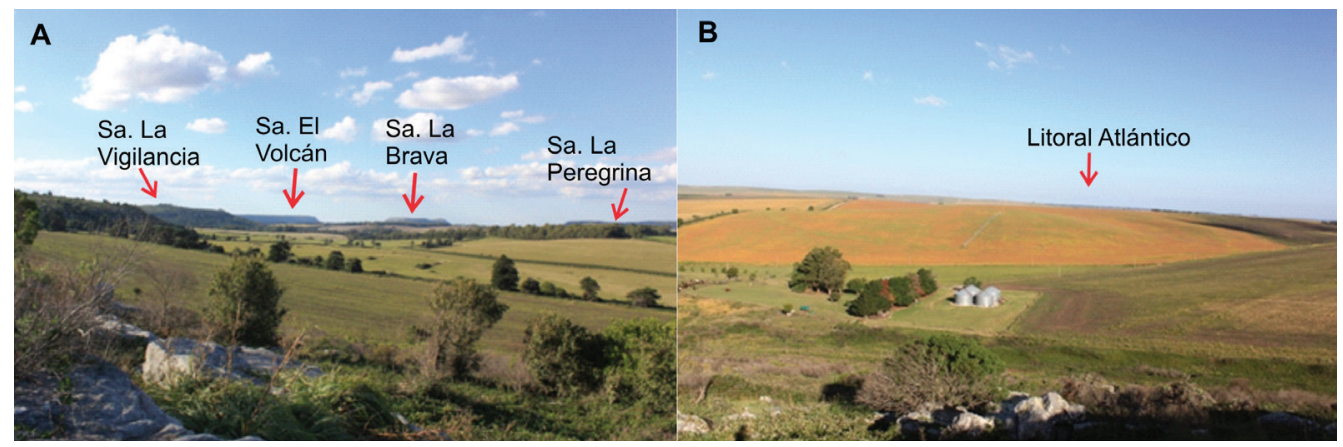

Figura 5. Visibilización territorial desde el Alero Molina I. A. Vista del paisaje noroeste delimitado por un horizonte de las principales sierras, B. Sector este y sudeste de llanura y lomadas que conducen al litoral marítimo

\section{AGRADECIMIENTOS}

Esta labor fue financiada por la ANPCyT mediante el PICT 2013 (1979) y recientemente con el PICT 2017 (1027), también se contó con el apoyo económico a través de dos subsidios (HUM 17/F546 y HUM 628/19) y la compra de un valioso equipo óptico otorgados por la Facultad de Humanidades (UNMDP). Destacamos la importante colaboración de Paolo Donadei Corada, Carlos Quintana y Caterina Quintana en las excavaciones del alero, también del geólogo Gustavo Adolfo Martínez por sus valiosas discusiones geoarqueológicas en el campo. Agradecemos a los estudiantes de la carrera de Historia (UNMDP) Jeremías Barroso y Ángel Bigarani por acompañar las actividades de campo durante la campaña 2018. Por último, agradecemos a los evaluadores Gustavo Martínez y anónimo cuyos importantes comentarios ampliaron nuestras reflexiones y mejoraron considerablemente este manuscrito.

\section{NOTAS}

1 La denominación de este sitio surgió en agradecimiento por la hospitalidad generosa que recibimos del encargado del puesto Sr. Molina y de su familia.

\section{BIBLIOGRAFÍA}

Bonnat, G. F.

2019. Raw material procurement and landscape use during the Pleistocene/Holocene transition in the eastern Tandilia Range (Buenos Aires, Argentina). PaleoAmerica 5 (1): 62-72.

2020. Análisis de la organización de la tecnología lítica de los grupos cazadores-recolectores tempranos del área de Tandilia Oriental (Buenos Aires). Sociedad Argentina de Antropología, Buenos Aires.

Bonomo, M. y A. Blasi

2010. Base regional de recursos líticos del Delta del Paraná. Estudio petrográfico de artefactos y afloramientos en el sur de Entre Ríos. Cazadores-Recolectores del Conos Sur. Revista de Arqueología 4: 17-41.

Bonomo, M. y L. Prates

2014. La explotación de depósitos secundarios de rodados en el curso medio del Río Negro y el litoral Marítimo Pampeano. En P. Escola y S. Hocsman (eds.), Artefactos líticos, movilidad y funcionalidad de Sitios en Sudamérica. Problemas y Perspectivas: 77-92. Oxford, BAR International Series 2628. 
D. L. Mazzanti y G. F. Bonnat - Alero Molina I. Funcionalidad y CRonología de un REPARo PEQUeÑo ...

Carbonari, J.

1972. Estratigrafía y Arqueología en la sierra Valdez, Partido de General Pueyrredón, Provincia de Buenos Aires. Ms Informe CIC-UNLP, La Plata.

Catella, L., A. Insaurralde y J. Angel

2020. Disponibilidad de fuentes de aprovisionamiento y selección de materias primas líticas en el sudoeste de la provincia de Buenos Aires. Comechingonia. Revista de Arqueología 24 (10): 1-29.

Colobig, M. M.

2011. Estudios paleobotánicos en sitios del borde oriental de Tandilia (Buenos Aires, Argentina). Pautas de aprovechamiento y uso de recursos vegetales en las sociedades de cazadores-recolectores. Tesis Doctoral inédita, Facultad de Humanidades y Arte. Universidad Nacional de Rosario, Rosario.

Criado Boado, $\mathrm{F}$.

1999. Del terreno al espacio: Planteamientos y perspectivas para la Arqueología del Paisaje. Criterios y Convenciones en Arqueología del Paisaje (CAPA) 6: 1-82. España.

Donadei Corada, J. P.

2020. Cazadores-recolectores del Holoceno medio en las sierras de Tandilia oriental (Argentina). Tecnología lítica, gestión de las materias primas y movilidad en el territorio. Tesis Doctoral inédita, Facultad de Filosofía y Letras, Universidad de Buenos Aires, Buenos Aires.

Fernández Osuna, M. A.

2019. Las Aves del Pleistoceno Tardío-Holoceno del borde oriental de Tandilia, Buenos Aires, Argentina: el caso especial de estudio del sitio Cueva Tixi. Tesis Doctoral inédita. Facultad de Ciencias Exactas, Físicas y Naturales, Universidad Nacional de Córdoba, Córdoba.

Flegenheimer, N., C. Bayón, M. Valente, J. Femeninas y J. Valente

2003. Long distance tool stone transport in the Argentine Pampas. Quaternary international 109-110: 49-64.

Hogg, A., T. Heaton, Q. Hua, J. Palmer, C. Turney, J. Southon, A. Bayliss, P. Blackwell, G. Boswijk, B.

Ramsey, C. Pearson, F. Petchey, P. Reimer, R. Reimer y L. Wacker

2020. SHCal20 Southern Hemisphere calibration, 0-55,000 years cal BP. Radiocarbon 62.

Loponte, D., P. Tchilinguirian y R. Sacur Silvestre

2010. Caracterización de afloramientos de calizas silicificadas de la provincia de Entre Ríos (Argentina) y su vinculación con los circuitos de abastecimiento prehispánico. En M. Feuillet, M. Terzaghi, B. Colasurdo, J. Sartori y S. Escudero (eds.), Avances y Perspectivas en la Arqueología del Nordeste: 125-139. Buenos Aires.

Martínez, G.

2006. Arqueología del curso medio del río Quequén Grande: estado actual y aportes a la arqueología de la región pampeana. Relaciones de la Sociedad Argentina de Antropología XXXI: 249-275.

Martínez, G. A.

2007. Procesos de formación de sitios en reparos rocosos de Tandilia. Cazadores-Recolectores del Cono Sur. Revista de Arqueología 3: 105-127.

Martínez, G. A., y D. L. Mazzanti

2017. Evidencia geoarqueológica de la transición Pleistoceno-Holoceno en reparos rocosos de Tandilia oriental (provincia de Buenos Aires). Relaciones de la Sociedad Argentina de Antropología XLII: 83-106.

Mazzanti, D. L.

2007. Arqueología de las relaciones interétnicas en las Sierras de Tandilia. Tesis Doctoral inédita, Facultad de Filosofía y Letras, Universidad de Buenos Aires, Buenos Aires. 
Relaciones de la Sociedad Argentina de Antropología 46 (1), enero-junio 2021: 363-372

2013. La arqueología de Tandilia en perspectiva histórica. Una revisión de sus aportes a la arqueología regional. Revista del Museo de La Plata, Sección Antropología 13 (87): 31-50.

Mazzanti, D. L. y G. F. Bonnat

2013. Paisajes arqueológicos y cazadores-recolectores de la transición Pleistoceno-Holoceno. Análisis de las cuencas de ocupación en Tandilia oriental, Provincia de Buenos Aires, Argentina. Relaciones de la Sociedad Argentina de Antropología, XVIII (2), 521-541.

Mazzanti, D. L., G. F. Bonnat, J. P. Donadei Corada y G. A. Martínez

2019. Cueva Alí Mustafá: un nuevo sitio arqueológico con ocupación temprana en las Sierras Orientales de Tandilia (Argentina). Arqueología 25 (2): 103-116.

Politis, G. y P. Madrid

2001. Arqueología Pampeana: estado actual y perspectivas. En E. Berberián y A. Nielsen (eds.), Historia Argentina Prehispánica: 737-814. Córdoba, Brujas.

Stuiver, M., P. J. Reimer y R.W. Reimer

2020. CALIB 7.1 Radiocarbon Calibration (program). http://calib.org.

Tapia, A.

1937. Las cavernas de Ojo de Agua y Las Hachas. Boletín de la Dirección de Minas y Geología 43, Buenos Aires. 